Handgelenk und Handwurzel

\section{Perkutane Verschraubung und Radius-Reizosteotomie zur Behandlung von Skaphoidpseudarthrosen}

Dedeoğlu SS et al. Results of percutaneous fixation and distal radius core decompression in scaphoid waist non-unions treated without grafting. Hand Surg Rehabil 2018; 37: $43-47$

Eine ausbleibende knöcherne Heilung (Non-Union) stellt nach Skaphoidfrakturen weiterhin ein bedeutsames Problem dar. Für diese Skaphoidpseudarthrosen ist eine ganze Reihe von Therapiemöglichkeiten beschrieben, wohl am häufigsten wird eine offene Rekonstruktion mit Einbringen eines Knochentransplantates und Osteosynthese durchgeführt.

Bei Frühstadien könnte aber eine andere, weniger invasive Möglichkeit infrage kommen, die türkische Mediziner untersucht haben. Dazu haben Dedeoğlu et al. zwischen Mai 2012 und September 2014 insgesamt 29 Patienten in eine prospektive Studie aufgenommen. Die Einschlusskriterien umfassten eine Skaphoidpseudarthrose nach Fraktur im mittleren Drittel mit minimal dislozierten Fragmenten, normalem skapholunärem Winkel ohne Humpback-Deformität und mit intaktem skapholunären Bandapparat.

Bei den Teilnehmern erfolgte zunächst die geschlossene Reposition unter Durchleuchtungskontrolle. Danach wurden von palmar eine 5-mm-Inzision über dem skaphotrapezialen Gelenk vorgenommen und die Kahnbeinfragmente mit einer kopflosen 3,5-mm-Kompressionsschraube (Länge im Mittel $26 \mathrm{~mm}$ ) fixiert. Im Anschluss führten die Operateure eine Radius-Reizosteotomie (radius core decompression) durch, bei der an der distalen Radiusmetaphyse nach Fensterung der Kortikalis die Spongiosa kürettiert wurde. Mit diesem Vorgehen sollte eine Verletzung simuliert werden, um eine Reaktion der umgebenden Blutgefäße zu induzieren und so die Durchblutung auch des Skaphoids zu verbessern. Postoperativ wurde das Gelenk in einem Unterarmgips über 8 - 10 Wochen ruhiggestellt, unmittelbar danach begannen Bewegungsübungen.

Für die jetzige Auswertung beurteilten die Mediziner

- das Eintreten einer knöchernen Heilung laut Standard-Röntgenaufnahmen und $\mathrm{CT}$,

- Schmerzen auf einer visuellen Analogskala (VAS) von 0 (keine Schmerzen) bis 10 (stärkste vorstellbare Schmerzen),

- die funktionellen Ergebnisse anhand des Mayo Wrist Score, bei dem Werte von 90 - 100 Punkte als ausgezeichnete Funktion gelten, 80 - 89 Punkte als gute Funktion, 65 - 79 Punkte als mäßige und $<65$ Punkte als schlechte Funktion,

- die Beweglichkeit im Handgelenk und - die grobe Kraft.

Unter den 29 Studienteilnehmern waren 27 Männer, das Durchschnittsalter betrug 29 Jahre. Zwischen der primären Verletzung und der jetzigen Operation lagen im Mittel 18 Monate, der Zeitraum reichte aber von 28-86 Wochen. Über eine durchschnittliche postoperative Beobachtungszeit von etwa 1,5 Jahren kam es bei 26 Patienten $(89,7 \%)$ zu einer vollständigen Heilung der Pseudarthrose, im Mittel nach 11 Wochen (Bereich 7-18 Wochen). Bei 3 Patienten wurde eine Revision erforderlich, bei der sekundär ein Knochentransplantat eingebracht wurde. Bei allen diesen Patienten hatte ursprünglich ein fortgeschrittener Befund (Pseudarthrose-Stadium III nach Slade und Geissler) vorgelegen. Weiterhin zeigten sich

- eine Schmerzminderung auf postoperativ 2 VAS-Punkte (0 - 7 Punkte) von 7 Punkten (6 - 9 Punkten) präoperativ,

- eine Verbesserung im Mayo Wrist Score auf 66 Punkte (20 - 90 Punkte) von präoperativ 36 Punkten

(15-65 Punkten),

- ein Bewegungsumfang

- für die Flexion von $61^{\circ}\left(35-80^{\circ}\right)$ gegenüber $47^{\circ}\left(30-60^{\circ}\right)$ präoperativ und

- für die Extension von $61^{\circ}\left(30-80^{\circ}\right)$ gegenüber $45^{\circ}\left(30-60^{\circ}\right)$ präoperativ sowie
- eine Zunahme der groben Kraft auf $32 \mathrm{~kg}$ von $22,4 \mathrm{~kg}$ präoperativ.

FAZIT

Bei Skaphoidpseudarthrosen in frühen Stadien kann bei sorgfältiger Auswahl der Patienten eine perkutane Verschraubung mit Radius-Reizosteotomie ohne zusätzliches Knochentransplantat erfolgreich sein, so die Autoren. Die Vorteile dieses Vorgehens sind die minimale Narbenbildung, die Vermeidung iatrogener Verletzungen von Bandapparat, Kapsel, Gefäßen und Nerven sowie ein geringes Infektionsrisiko. Nicht zuletzt besteht damit keine Gefahr von Komplikationen im Bereich einer Knochenspan-Entnahmestelle.

Dr. Elke Ruchalla, Bad Dürrheim

\section{Kommentar}

Die Autoren behandelten 29 Kahnbeinpseudarthrosen im mittleren Drittel ohne begleitende Achsenfehlstellung (DISI, Humpback) bei im MRT nachgewiesener Durchblutung des proximalen Fragmentes mit durchschnittlichem Abstand von 18 Monaten zur Fraktur. Mit minimalinvasiv eingebrachter Doppelgewindeschraube von palmar und begleitender Core-Dekompression in der Radiusmetaphyse erzielten sie eine Durchbauung in 26 Fällen (90\%).

Auch wenn keine Vergleichsgruppe vorliegt, wurde eine Erfolgsquote erreicht, die im Bereich der klassischen Methode Beckenkamm mit Schraube - in der Literatur bei vergleichbarer Ausgangslage berichtet wird. Welche zusätzliche Rolle die Core-Dekompression spielt, ist schwer einzuschätzen, aber die Arbeit macht deutlich, dass offensichtlich bei entsprechenden Vorbedingungen mit fehlender Achsenfehlstellung und erhaltener proximaler Durchblutung ein offenes Vorgehen nicht zwingend erforderlich ist, was auch durch andere Publikationen bestätigt wird. Bei abgedeckelter sklerosierter Pseudarthrose ist diese Vorgehensweise kontraindiziert, würde sich aber anhand des MRT mit verminderter 
oder aufgehobener Druchblutung analysieren lassen. Zusammenfassend zeigt die Arbeit, dass bei nur geringer Dislokation, fehlender Achsenfehlstellung und erhaltener proximaler Durchblutung proximal ein minimalinvasives Vorgehen ohne Spongiosaplastik zur Anwendung kommen kann. Die Erfolge der hochenergetischen Stoßwellentherapie in der Behandlung der Kahnbeinpseudarthrose können zusätzlich als Beleg für ein geschlossenes Vorgehen gewertet werden. Welche Rolle die Core-Dekompression dabei einnimmt, muss durch weitere Studien geklärt werden.
Autorinnen/Autoren

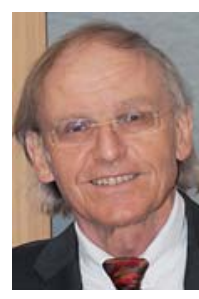

Prof. Dr. Hermann Krimmer,

Zentrum für Hand- und

Fußchirurgie, Krankenhaus

St. Elisabeth, Ravensburg 